\title{
Emerging role of SIRT2 in non-small cell lung cancer (Review)
}

\author{
MENGGE ZHENG, CHANGYONG HU, MENG WU and YUE EUGENE CHIN
}

Institute of Biology and Medical Sciences, Soochow University Medical College, Suzhou, Jiangsu 215123, P.R. China

Received October 31, 2020; Accepted April 16, 2021

DOI: 10.3892/ol.2021.12992

\begin{abstract}
Non-small cell lung cancer (NSCLC) is one of the most devastating cancer types, accounting for $>80 \%$ of lung cancer cases. The median relative survival time of patients with NSCLC is $<1$ year. Lysine acetylation is a major post-translational modification that is required for various biological processes, and abnormal protein acetylation is associated with various diseases, including NSCLC. Protein deacetylases are currently considered cancer permissive partly due to malignant cells being sensitive to deacetylase inhibition. Sirtuin 2 (SIRT2), a primarily cytosolic nicotinamide adenine dinucleotide-dependent class III protein deacetylase, has been shown to catalyze the removal of acetyl groups from a wide range of proteins, including tubulin, ribonucleotide reductase regulatory subunit M2 and glucose-6-phosphate dehydrogenase. In addition, SIRT2 is also known to possess lysine fatty deacylation activity. Physiologically, SIRT2 serves as a regulator of the cell cycle and of cellular metabolism. It has been shown to play important roles in proliferation, migration and invasion during carcinogenesis. It is notable that both oncogenic and tumor suppressive functions of SIRT2 have been described in NSCLC and other cancer types, suggesting a context-specific role of SIRT2 in cancer progression. In addition, inhibition of SIRT2 exhibits a broad anticancer effect, indicating its potential as a therapeutic for NSCLC tumors with high expression of SIRT2. However, due to the diverse molecular and genetic
\end{abstract}

Correspondence to: Professor Yue Eugene Chin, Institute of Biology and Medical Sciences, Soochow University Medical College, 199 Ren'ai Road, Suzhou, Jiangsu 215123, P.R. China

E-mail: chinyue@suda.edu.cn

Abbreviations: NSCLC, non-small cell lung cancer; $\mathrm{NAD}^{+}$, nicotinamide adenine dinucleotide; SIRT2, Sirtuin 2; Sir2, silent information regulator 2; ADP, adenosine diphosphate; EGFR, epidermal growth factor receptor; SIRT1, silent information regulator homolog 1; AKR1C1, aldo-keto reductase family 1 member C1; RalB, Ras-like proto-oncogene B; AGK2, 2-cyano-3[5-(2,5-dichlorophenyl)-2-furanyl]-N-5-quinolinyl-2-propenamide; NES, nuclear export signal; ACLY, ATP-citrate lyase; CSC, cancer stem cell; ESC, embryonic stem cell; PGAM, phosphoglycerate mutase; TIL, tumor-infiltrating lymphocyte

Key words: sirtuin 2, NSCLC, function, pathogenic mechanism, therapeutic target characteristics of NSCLC, the context-specific function of SIRT2 remains to be determined. The current review investigated the functions of SIRT2 during NSCLC progression with regard to its regulation of metabolism, stem cell-like features and autophagy.

\section{Contents}

1. Introduction

2. SIRT2 gene and protein

3. SIRT2 biological functions

4. SIRT2 function in various tumor types

5. Emerging roles of SIRT2 in NSCLC

6. SIRT2 targeting therapeutic strategy

7. Conclusion

\section{Introduction}

The Sirtuin 2 (SIRT2) gene was initially identified as ribonuclease mar1, which acted by preventing the expression of mating genes in yeast (1). The yeast silent information regulator 2 (SIR2) was shown to be involved in transcriptional silencing, ribosomal DNA recombination, life span and other physiological functions (2-4). It was initially hypothesized that SIR2 possessed an adenosine diphosphate (ADP)-ribosyltransferase activity that could transfer ADP-ribose from nicotinamide adenine dinucleotide (NAD) to histones (5). This activity was subsequently shown to be a low efficiency side reaction (6). It is well accepted that SIR2 is a NAD-dependent lysine deacetylase. The SIRT enzymes are highly conserved from bacteria to humans. Bacteria and archaea only express one or two SIRT, while mammals have seven SIRT homologs (SIRT1-7) $(7,8)$. The seven mammalian SIRT have different subcellular localization patterns, including cytoplasmic (SIRT1 and 2), nuclear (SIRT1, 2, 3, 6 and 7) and mitochondrial (SIRT3, 4 and 5). Among them, SIRT1-3 belong to class I SIRTs and have higher homology to the yeast silent information regulator 2 (Sir2), histone deacetylase (Hst)1 and Hst2. All these enzymes exhibit potent deacetylase activity (7).

SIRT2 is a member of the SIRT family and is also known as SIR2, SIRT type 2 or Sir2-related protein type 2; it belongs to the classic type III deacetylases and acts in a NAD ${ }^{+}$dependent manner (9). SIRT2 is unique amongst SIRTs as it is the only primary cytoplasmic enzyme with robust deacetylase activity. Recently, SIRT2 has been shown to catalyze the removal of 
lysine fatty acylation, including hexanoylation, decanoylation and myristoylation (10). The newly characterized de-acylation activities of SIRT2 have improved the current knowledge on the function of this enzyme and have provided novel opportunities to study the physiological and pathological role of SIRT2.

\section{SIRT2 gene and protein}

The human SIRT2 gene is located on chromosome 19 with 16 exons spanning 21 kilobases of the genomic DNA. The SIRT2 transcript undergoes alternative splicing and produces three isoforms with different cellular and tissue distributions, and different functions $(11,12)$. The full-length isoform 1 is abundant in the skeletal muscle, while isoform 2, which lacks the N-terminal 37 residue, accumulates in the brain. Both isoforms 1 and 2 are enzymatically active and are able to shuttle between the nucleus and the cytoplasm. In contrast to these findings, isoform 5 lacks a nuclear export signal that contains the entire nuclear export signal (NES) and a short fragment of the catalytic domain, resulting in the nuclear enrichment and loss of catalytic activity (12).

$\mathrm{X}$-ray crystallographic studies have revealed that the human SIRT family enzymes contain two major functional domains: A small domain that binds to zinc ions and a large domain that is responsible for $\mathrm{NAD}^{+}$binding. A conserved large substrate binding groove is also presented at the interface of the two domains (13). The longest SIRT2 isoform contains 389 amino acids, of which the 65-340 amino acid sequence is the NAD-dependent catalytic domain (14). The 41-51 amino acid sequence at the N-terminal of SIRT2 is a NES, which is responsible for guiding the cytoplasmic localization of the protein (15). The detailed schematic diagram of each domain of the SIRTs family is shown in Fig. 1. Despite their conserved structural features, human SIRTs diverge significantly in their subcellular distribution, substrate targets and enzymatic activity characteristics. For example, the lipoamidase activity of SIRT4 is superior to its deacetylase activity (16). SIRT6 exhibits preferential activity for the removal of long chain fatty acyls (10). SIRT7 employs nuclear acid oligos (double-stranded DNA, ribosomal RNA and transfer RNA) as co-factors for catalysis (17). SIRT1, SIRT2 and SIRT3 possess a robust deacetylase activity.

\section{SIRT2 biological functions}

Although SIRT2 is predominantly cytoplasmic, it shuttles into the nucleus under certain circumstances, such as mitosis or bacterial infection (18). SIRT2 predominantly regulates cellular processes through its enzymatic activity (18-23). Previous studies indicated a conserved role of SIR2 in extending the lifespan of yeast, flies and worms in a deacetylase-dependent manner (24-26). In mammals, the significant longevity gene BUB1 mitotic checkpoint serine/threonine kinase B (BubR1) was shown to be deacetylated by SIRT2. The latter deacetylates BubR1 at the K668 site, thereby stabilizing the BubR1 protein. As a consequence, the lifespan of progeroid hypomorphic BubR1 mice was largely increased by SIRT2 overexpression (27). In general, the anti-aging effect of SIRT2 seems to be conservative from yeast to mammals.
Mammalian SIRT2 has been shown to restrain cell cycle progression in a deacetylase activity-dependent manner (28). SIRT2 is associated with chromatin during mitosis and facilitates chromatin condensation by deacetylating Histone $\mathrm{H} 4$ lysine (H4K) 16 (29). The deacetylation of H4K16 is in turn essential for the mitotic deposition of H4K20 methylation (30). The upregulation of the level of mitotic regulators in SIRT2-knockout mice confirmed the cell cycle regulatory function of SIRT2 (31). It was proposed that SIRT2 regulates mitosis by deacetylating cadherin-1 and cell division cycle protein 20 homolog, which are two adenomatous polyposis coli/C coactivators (31). In addition to its action as a mitotic regulator, SIRT2 also deacetylates ribonucleotide reductase regulatory subunit M2 at the K95 site during the S phase of the cell cycle. This deacetylation increases the dNTP pool size and accelerates DNA replication fork progression by enhancing ribonucleotide reductase activity, which ultimately results in an increased cancer cell proliferative rate (32).

A number of previous studies have shown that SIRT2 also plays a role in regulating cell metabolism $(33,34)$. SIRT2 can promote the metastasis of gastric cancer through the RAS/ERK/JNK/matrix metalloproteinase-9 pathway by increasing phosphoenolpyruvate carboxykinase 1-associated metabolism (35). SIRT2 promotes glycolysis and tumor growth by deacetylating the K305 site of pyruvate kinase M2, a hallmark enzyme that bridges metabolism and immunity (36). A comprehensive understanding of the function and mechanism of SIRT2 in the progression of various cancer types is imminent. Clinical data and mechanisms of action for SIRT2 in cancer are shown in Table I. Glucose-6-phosphate dehydrogenase (G6PD) is a key enzyme that is involved in the pentose phosphate pathway and is responsible for producing NADPH. Deacetylation of the residue K403 of G6PD by SIRT2 increases its activity and enhances NADPH production (37). It is important to note that SIRT2 can inhibit glycolysis and metabolic reprogramming of induced pluripotent stem cells (iPSCs) by deacetylating the following four key glycolytic enzymes: Aldolase, phosphoglycerate kinase 1, enolase and GAPDH (19). A recent study demonstrated that SIRT2 is a master organizer of T-cell metabolism, since it inhibits T-cell glycolysis and impairs T-cell effector functions by deacetylating a number of metabolic enzymes, such as phosphofructokinase, $\alpha$-ketoglutarate dehydrogenase, succinate dehydrogenase complex, subunit A, flavoprotein variant and succinyl-CoA ligase (GDP-forming) subunit $\alpha$ mitochondrial (38).

In addition to its classical deacetylase activity, SIRT2 has also been shown to catalyze the removal of long chain fatty acyls from Kras4a and Ras like proto-oncogene B (RalB) $(39,40)$. Furthermore, the de-myristoylation activity of SIRT2 towards ADP-ribosylation factor 6 lysine 3 was recently identified (41). The diverse functions of SIRT2 in the nervous system, mitosis, genome integrity, cell differentiation, cell homeostasis, aging, infection, inflammation, oxidative stress and autophagy have been previously reviewed (42-44).

\section{SIRT2 function in various tumor types}

Dysregulation of SIRT2 in cases of gene amplification and mutation, protein overexpression and mislocalization 


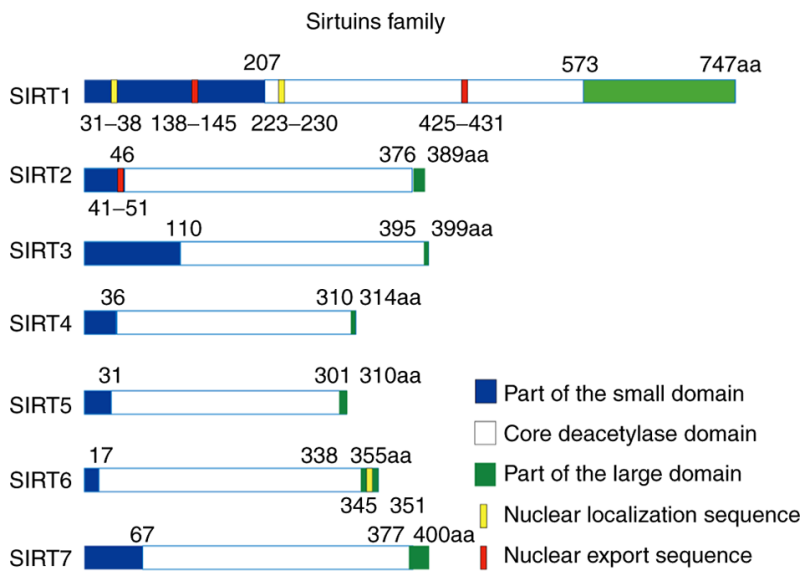

Figure 1. Detailed schematic diagram of each domain of the SIRTs family SIRTs 1-7 have numerous types of isoforms, but only the longest isoforms are shown in this figure. SIRT, sirtuin.

has been associated with the progression of various cancer types. Cross-cancer analysis of The Cancer Genome Atlas database indicates that the SIRT2 gene is amplified in $~ 9 \%$ (52 out of 584 cases) of ovarian epithelial tumors and 4\% (41 out of 1,053 cases) of NSCLCs (https://www.cancer.gov/about-nci/organization/ccg/research/structural-genomics/tcga). The gene locus of SIRT2 is known to be frequently deleted in human oligodendrogliomas (45). In addition, a number of somatic mutations within SIRT2 are found in endometrial carcinoma, melanoma, leukemia and NSCLC (cbioportal website) (http://www. cbioportal.org/). Particularly, multiple cancer-associated SIRT2 mutations at evolutionarily conserved sites have been reported as functionally significant (46). For example, the R42L mutation found in lung cancer decreased the protein levels of SIRT2, while the P128L mutation found in both colon and uterine cancer types abrogated the enzymatic activity of SIRT2 (46).

Due to tumor heterogeneity, the SIRT2 expression pattern is not always consistent. According to two recent review articles, SIRT2 expression was elevated in neuroblastoma, uveal melanoma, renal cell carcinoma and acute myeloid leukemia, while it was decreased in glioma, neck squamous cell carcinoma, breast cancer, prostate cancer and liver cancer $(47,48)$. In addition, dual SIRT2 expression patterns have been noted in NSCLC. Li et al (49) demonstrated that SIRT2 mRNA and protein expression levels were downregulated in NSCLC. Grbesa et al (50) demonstrated that the expression levels of the SIRT2 proteins were significantly higher in lung primary tumors than those noted in normal tissues. It is notable that Gao et al (51) demonstrated patients with NSCLC with low SIRT2 expression had longer overall survival (OS) compared with those with high SIRT2 expression, according to a survival analysis of 1,926 patients and SIRT2 expression levels were significantly related to the survival time of patients with lung adenocarcinoma (ADC) but not squamous cell carcinoma (SCC). Despite nuclear and cytoplasmic shuttling, SIRT2 is in fact a primary cytoplasmic protein. High levels of mislocalized nuclear SIRT2 protein were associated with shorter disease-free survival time in ER-negative breast cancer (52). Gong et al (53) revealed that the combination of SIRT1 and
SIRT2 was an improved recurrence-free survival prediction model for NSCLC. It is also notable that SIRT2 has been identified as a candidate plasma biomarker in invasive cervical cancer (54).

The role of SIRT2 in cancer is controversial. SIRT2 has been reported to exert either tumor suppressor or oncogenic functions. It was initially proposed as a tumor suppressor due to its regulatory role on the mitotic checkpoint and due to its deacetylase activity on histone $\mathrm{H} 3 \mathrm{~K} 56$, which is a frequent modification noted in cancer cells $(55,56)$. The tumor suppressive role of SIRT2 was supported by genetic experiments demonstrating that Sirt2-deficient mice exhibited increased tumor incidence (30). In contrast to these observations, the tumor promoting activity of SIRT2 was supported by its ability to deacetylate p53 and downregulate its transcriptional activity (57). Moreover, SIRT2 deacetylates K5 of lactate dehydrogenase A and increases its activity and protein levels, thereby accelerating glycolysis and lactate production, which in turn leads to increased cancer cell proliferation and migration (58). Moreover, SIRT2 inhibitors have been shown to have broad anticancer activity $(38,59)$, suggesting their therapeutic potential in cancer cells.

These seemingly opposite observations may reflect a context-specific role of SIRT2 in cancer progression. Researchers are therefore encouraged to appropriately assess the clinical and molecular features in order to determine SIRT2 protein abnormalities in NSCLC.

\section{Emerging roles of SIRT2 in NSCLC}

Cancer-promoting effect of SIRT2 in NSCLC. Kras and epidermal growth factor receptor (EGFR) mutations, and Myc amplification are among the most common molecular abnormalities in NSCLC. SIRT2 positively regulates Kras activity by catalyzing deacetylation of Kras-K104 or fatty deacylation of K-Ras4a (39,60). The SIRT2 inhibitor JH-T4 has been described as a potent anticancer agent. The mode of action of JH-T4 possibly involves increased fatty acylation of K-Ras4a (61). In contrast to this evidence, in previous studies, SIRT2 stabilized Myc oncoprotein by repressing neuronally expressed developmentally downregulated 4 (NEDD4) E3 ubiquitin-protein ligase gene expression (62). In addition, Kras-mutant NSCLC cells were sensitive to loss of SIRT2 expression (60). This is consistent with the fact that degradation of SIRT2 is positively correlated with NSCLC cell proliferation (63).

In addition, SIRT 2 can participate in cancer progression by regulating physiological processes, including metabolism and autophagy. Enhanced glycolysis is a distinctive and prominent feature of cancer cells. Phosphoglycerate mutase (PGAM) is a glycolytic enzyme that catalyzes the reversible conversion of 3-phosphoglycerate to 2-phosphoglycerate. PGAM is considered oncogenic, while its inhibition attenuates tumor growth in NSCLC cells (64). SIRT2 deacetylates the K100 residue of PGAM and facilitates its activation, resulting in enhanced NADPH production and accelerated tumor growth (64). The autophagic pathway is associated with tumor suppression. Forkhead box protein O1 (FoxO1) is involved in the induction of autophagy and serves as a tumor suppressor, ectopically expressed FoxO1 interacts with SIRT2 and is deacetylated by SIRT2 in H1299 NSCLC cells (65). In either lung or colon 
Table I. Clinical data and mechanisms of action of SIRT2 in cancer.

\begin{tabular}{|c|c|c|c|c|}
\hline $\begin{array}{l}\text { Cancer type } \\
\text { or cell line }\end{array}$ & Clinical data & Effect of SIRT2 & Mechanisms of action & Authors (Refs.) \\
\hline NSCLC & $\begin{array}{l}\text { Poor prognosis } \\
\text { shorter } \\
\text { RFS or OS times }\end{array}$ & $\begin{array}{l}\text { Protumorigenic role in } \\
\text { lung cancer SIRT2 } \\
\text { proteins can }\end{array}$ & $\begin{array}{l}\text { Combination of highly expressed SIRT1 } \\
\text { and predict shorter RFS or OS times in } \\
\text { patients with NSCLC }\end{array}$ & Grbesa et al (50) \\
\hline $\begin{array}{l}\text { Gastric } \\
\text { cancer }\end{array}$ & & $\begin{array}{l}\text { Promotes migrationand } \\
\text { invasion of gastric cancer }\end{array}$ & Regulates PEPCK1-related metabolism & Li et al (35) \\
\hline $\begin{array}{l}\text { Mammary } \\
\text { tumor cells }\end{array}$ & & $\begin{array}{l}\text { Regulates tumor growth } \\
\text { and glycolysis }\end{array}$ & Deacetylates the K305 site of PKM2 & Park et al (36) \\
\hline $\begin{array}{l}\text { Colorectal } \\
\text { cancer }\end{array}$ & Poor prognosis & Promotes tumorigenesis & $\begin{array}{l}\text { Activating the STAT3/VEGFA signaling } \\
\text { pathway }\end{array}$ & Hu et al (80) \\
\hline HEK293T & & Promotes tumorigenesis & $\begin{array}{l}\text { Sirt2 interacts with } 14-3-3 \beta / \gamma \text { and } \\
\text { downregulates transcriptional activity of p53 }\end{array}$ & Jin et al (57) \\
\hline $\begin{array}{l}\text { Pancreatic } \\
\text { cancer }\end{array}$ & & $\begin{array}{l}\text { Promote proliferation and } \\
\text { migration }\end{array}$ & $\begin{array}{l}\text { Accelerates glycolysis and lactate } \\
\text { production by deacetylating K5 of LDH-A } \\
\text { to increases its activity and protein level }\end{array}$ & Zhao et al (58) \\
\hline $\begin{array}{l}\text { Neuroblast } \\
\text { oma cell } \\
\text { pancreatic } \\
\text { cancer }\end{array}$ & & Pro-cancer effect & $\begin{array}{l}\text { Stabilizes Myc oncoproteins by repressing } \\
\text { NEDD4 expression }\end{array}$ & Liu et al (62) \\
\hline NSCLC & & Pro-cancer effect & $\begin{array}{l}\text { SPOP inhibits NSCLC cell growth by } \\
\text { promoting SIRT } 2 \text { degradation }\end{array}$ & Luo et al (63) \\
\hline NSCLC & $\begin{array}{l}\text { Poor prognosis, } \\
\text { shorter OS times }\end{array}$ & Pro-cancer effect & $\begin{array}{l}\text { OS time of NSCLC patients with low SIRT2 } \\
\text { expression levels was significantly higher } \\
\text { compared with high SIRT2 expression levels }\end{array}$ & Gao et al (51) \\
\hline NSCLC & & $\begin{array}{l}\text { Suppresses metastasis of } \\
\text { NSCLC }\end{array}$ & $\begin{array}{l}\text { Inhibits transcriptional activity of Stat } 3 \text { by } \\
\text { abrogating AKR } 1 \mathrm{C} 1-\text { Stat } 3 \text { binding }\end{array}$ & Zhu et al (68) \\
\hline NSCLC & & Anticancer effect & $\begin{array}{l}\text { Attenuates the oncogenic activity of ACLY } \\
\text { by acting as the primary deacetylase of } \\
\text { ACLY in NSCLC cells }\end{array}$ & Lin et al (69) \\
\hline $\begin{array}{l}\text { Lung } \\
\text { cancer }\end{array}$ & & Anticancer effect & $\begin{array}{l}\text { Inhibits migration of lung cancer A549 } \\
\text { cells by the removal of long chain fatty } \\
\text { acyls from RalB protein }\end{array}$ & Spiegelman et al (40) \\
\hline NSCLC & & Anticancer effect & $\begin{array}{l}\text { Overexpression of SIRT2 in lung cancer } \\
\text { cell lines induces cell apoptosis induction, } \\
\text { cell cycle arrest and cell proliferation } \\
\text { inhibition by deacetylating Skp2 degraded }\end{array}$ & Li et al (49) \\
\hline NSCLC & & Anticancer effect & $\begin{array}{l}\text { SIRT2 is readily in NSCLC through HRD- } \\
\text { mediated ubiquitination }\end{array}$ & Liu et al (67) \\
\hline $\begin{array}{l}\text { Breast } \\
\text { cancer }\end{array}$ & $\begin{array}{l}\text { Poor prognosis } \\
\text { of breast CSCs }\end{array}$ & Promote differentiation & $\begin{array}{l}\text { NOTCH signaling pathway induces } \\
\text { SIRT2 to deacetylate ALDH1A1 }\end{array}$ & Zhao et al (75) \\
\hline
\end{tabular}

SIRT, sirtuin; NSCLC, non-small cell lung cancer; OS, overall survival; CSC, cancer stem cell; RalB, Ras like proto-oncogene B; ACLY, ATP-citrate lyase; AKR1C1, aldo-keto reductase family 1 member C1; SPOP, speckle type BTB/POZ protein; RFS, recurrence-free survival; PEPCK1, phosphoenolpyruvate carboxykinase 1; PKM2, pyruvate kinase M2 isoform; LDH-A, lactate dehydrogenase A; NEDD4, neuronally expressed developmentally downregulated 4; Skp2, S phase kinase-associated protein 2.

cancer cells, inhibition of SIRT2 increases FoxO1 acetylation and promotes the interaction between FoxO1 and autophagy related 7 , which is required for the induction of autophagy, a process that is negatively correlated to tumor development (65). Tang et al (66) revealed that the high expression of
SIRT2 contributes to induce the protective autophagy mechanism of HL-60/A cells, which is closely related with the drug resistance of patients.

In addition, a clinical survival analysis of 1,926 patients with NSCLC demonstrated that the median survival time of 


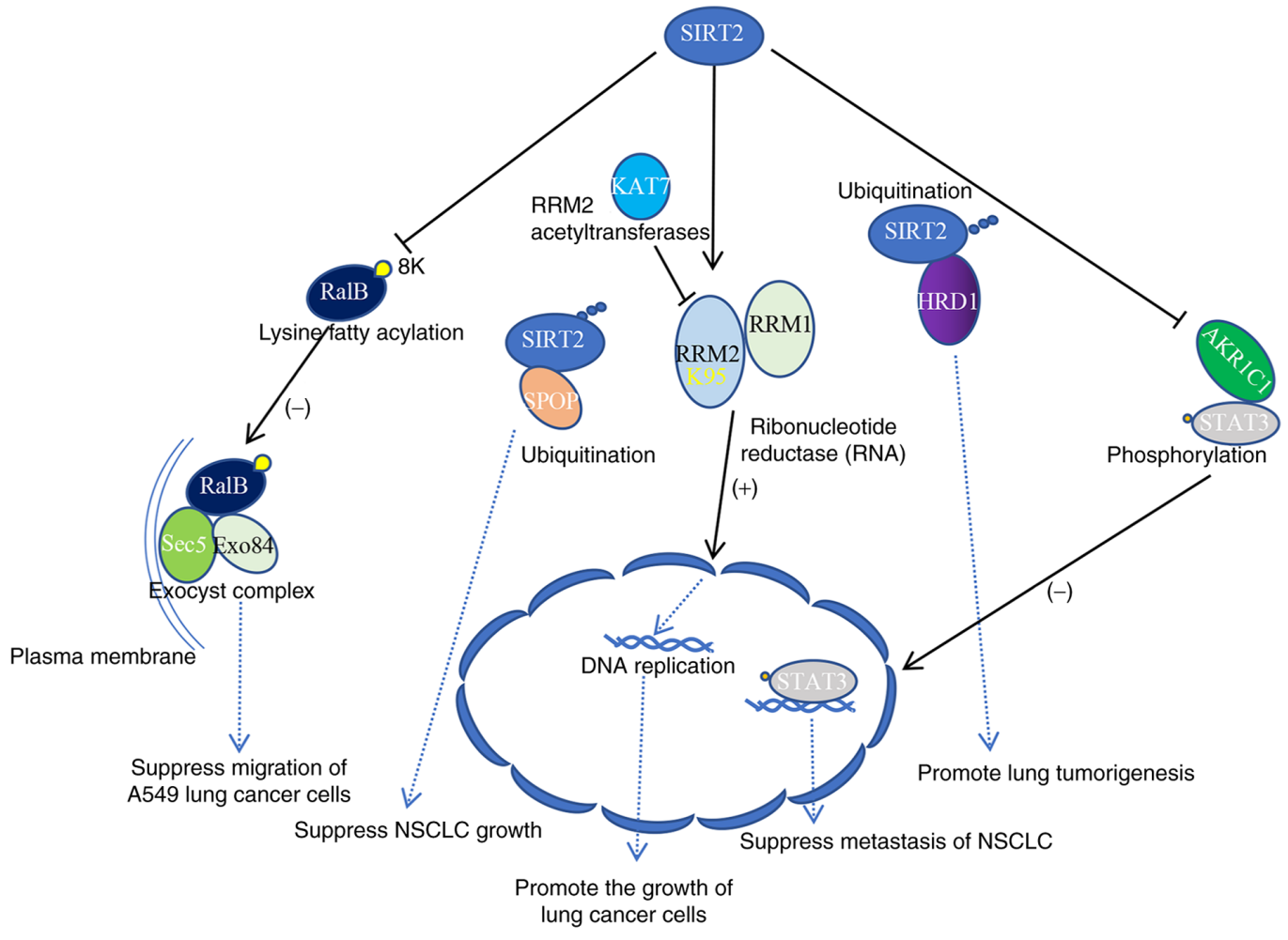

Figure 2. Schematic diagram of the mechanism of action of SIRT2 in NSCLC. RalB lysine fatty acylation promotes membrane localization and A549 cell migration. SIRT2 can significantly inhibit the formation and plasma membrane localization of the RalB-Sec5-Exo84 exocyst complex by regulating RalB8K lysine fatty acylation. Therefore, SIRT2 suppresses migration of A549 lung cancer cells by downregulating RalB8K lysine fatty acylation. SPOP can bind to SIRT2 and mediate its degradation by the proteasome. Subsequently, it inhibits the growth of NSCLC. By deacetylating the RRM2 K95 subunit of RNR, SIRT2 contributes to dNTP synthesis and DNA replication, thereby promoting the growth of lung cancer cells. The E3 ubiquitin ligase HRD1 binds to SIRT2 and promotes its degradation, which hinders the anticancer effect of SIRT2. By deacetylating AKR1C1, SIRT2 attenuates AKR1C1-STAT3 binding and STAT3 phosphorylation, thereby inhibiting the transcriptional activity of STAT3 target genes. SIRT2, sirtuin 2; NSCLC, non-small cell lung cancer; RalB, Ras-like proto-oncogene B; SPOP, speckle type BTB/POZ protein; RRM2, ribonucleotide reductase regulatory subunit M2; RNR, ribonucleotide reductase; HRD1, E3-ubiquitin protein ligase HRD1; AKR1C1, aldo-keto reductase family 1 member C1.

patients with low SIRT2 expression levels was significantly higher than that of patients with high SIRT2 expression levels (15.0 versus 14.0 months, $\mathrm{P}=0.029)$ (51). This evidence supports an oncogenic role of SIRT2 in NSCLC.

Anticancer effects of SIRT2 in NSCLC.Conflicting studies have nevertheless shown that SIRT2 expression is downregulated in NSCLC and that SIRT2 can inhibit tumor growth $(49,67)$. Zhu et al (68) reported that deacetylation of aldo-keto reductase family 1 member C1 (AKR1C1) by SIRT2 inhibited the binding of AKR1C1 to STAT3, therefore decreasing the transcriptional activity of STAT3 and inhibiting migration of NSCLC cells. SIRT2 was shown to inhibit migration of A549 lung cancer cell by the removal of fatty acyls from the RalB protein (40). Similarly, overexpression of SIRT2 in A549 and H1299 cells caused inhibition of cell proliferation, induction of cell apoptosis and cell cycle arrest by deacetylating S-phase kinase-associated protein 2 (49).

Increased lipogenesis plays a critical role in tumor growth. ATP-citrate lyase (ACLY) exhibits an oncogenic function in NSCLC. Acetylation of K540, K546 and K554 (3K) residues on ACLY inhibits its ubiquitylation and degradation, ACLY is a key enzyme that catalyzes the ATP-dependent conversion of citrate and coenzyme A (CoA) to oxaloacetate and acetyl-CoA, SIRT2 deacetylates ACLY and promotes its degradation, leading to decreased fatty acid synthesis, as well as delayed tumor growth in NSCLC cells (69). SIRT2 attenuates the oncogenic activity of ACLY by acting as its primary deacetylase in NSCLC cells (69). In addition, Mu et al (70) revealed that SIRT1/2 inhibition triggers pro-survival autophagy by increasing acetylation of HSPA5 and upregulating expression levels of ATF4 and DDIT4 to obstruct the mTOR signaling pathway in human NSCLC cells. SIRT2 directly binds to transcription factor EB, to regulate acute shear stress-induced cell apoptosis by regulating the release of autophagy components and exosomes, which contributes to the suppression of tumorigenesis and the metastasis of NSCLC (71). A recent study demonstrated that SIRT2 was readily degraded via homologous recombination repair-mediated ubiquitination in NSCLC (67), supporting the anticancer function of SIRT2 in this disease.

Taken together, the data demonstrate that SIRT2 can participate in the occurrence and development of various cancer types by regulating a variety of physiological processes; it has different mechanisms of action in various cancer types, and a schematic diagram of its mechanism of action in NSCLC is shown in Fig. 2.

\section{SIRT2 targeting therapeutic strategy}

SIRT2 and drug resistance. EGFR-activating mutations are noted in $\sim 20 \%$ patients with NSCLC. EGFR tyrosine kinase 
inhibitors are currently the standard treatment for patients with NSCLC and EGFR mutations. However, drug resistance is a major factor affecting the efficacy of anticancer therapy. Using large-scale screening, Bajpe et al (72) demonstrated that loss of SIRT2 conferred resistance to EGFR inhibitors in NSCLC and colon cancer. SIRT2 deacetylates MEK1 and inhibits its activation. Since loss of SIRT2 results in increased levels of MEK1 acetylation and phosphorylation, the increase in MEK1 activation and downstream ERK phosphorylation may lead to cancer recurrence. Similarly, SIRT2 loss conferred resistance to the effects of BRAF and MEK inhibitors in BRAF-mutant melanoma and Kras-mutant colon cancer, respectively (72). In contrast to these findings, an increase in SIRT2 expression levels may cause multidrug resistance in acute myelogenous leukemia by activating the ERK1/2 signaling pathway (73). In addition, SIRT2 exhibited protective effects against chemotherapy-induced peripheral neuropathy in a subcutaneous lung cancer mouse model. This condition is one of the most common causes of chemotherapy dose reduction and discontinuation. Cisplatin induces SIRT2 nuclear accumulation in dorsal root ganglia neurons, allowing SIRT2 to participate in the repair of cisplatin-generated DNA damage (74). Multiple studies have shown that SIRT2 contributes to the stemness of cancer stem cells (CSCs), which provides a further link between SIRT2 and chemoresistance in $\operatorname{NSCLC}(75,76)$.

SIRT2 and cancer stem cells. CSCs are considered the 'seeds' of cancer cells, with self-renewal ability and multilineage differentiation potential. CSCs are resistant to radiotherapy and chemotherapy, and are closely associated with tumor recurrence and metastasis (77). It has been shown that SIRT2 is involved in regulating the stemness of embryonic stem cells (ESCs) and CSCs. SIRT2 expression is significantly downregulated in both human ESCs and human pluripotent stem cells, while its upregulation is noted during mouse ESC differentiation. In addition, a study showed that depletion of SIRT2 prominently increased iPSC generation, while overexpression of SIRT2 significantly reduced this process (19). These effects are mainly attributed to the deacetylation and inactivation of glycolytic enzymes by SIRT2; Cha et al (19) reported that downregulation of SIRT2 by miR-200c promotes glycolysis and acetylation of glycolytic enzymes, which contributes to cellular reprogramming of human PSCs. Aldehyde dehydrogenase 1A1 (ALDH1A1) serves as a marker of CSCs in NSCLC and participates in their maintenance (78). ALDH1A1 activity is inhibited by K353 acetylation, which can be further deacetylated by SIRT2. Activation of the NOTCH signaling pathway induces deacetylation of ALDH1A1, which is catalyzed by SIRT2. This leads to activation of ALDH1A1 and increased self-renewal properties of CSCs (75). Similar pro-self-renewal effects of SIRT2 have been demonstrated in renal cell carcinoma CSCs; Wei et al (76) reported that SIRT2 may be highly expressed in the RCC stem-like cells and that it contributes to cancer metastasis. In addition, it was reported that the SIRT2-selective inhibitor 2-cyano-3-(5-(2, 5-dichlorophenyl)-2-furanyl)-N-5-quinolinyl-2-propenamide (AGK2) showed the most potent antiproliferative effect in glioblastoma multiforme CSCs (79).

SIRT2 in cancer immunotherapy. Tumor infiltrating lymphocyte (TIL) therapy is considered a promising option for treating

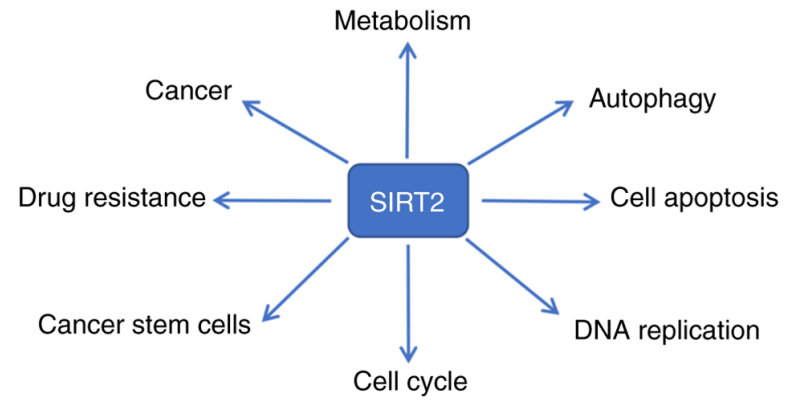

Figure 3. A schematic diagram of the biological functions of SIRT2. SIRT2, sirtuin 2 .

patients with metastatic NSCLC, whereas loss of T-cell effector functions within the tumor microenvironment can limit the clinical efficacy of this therapeutic method. SIRT2 has been suggested as a master regulator of T-cell metabolism and an immune checkpoint in TILs (38). By utilizing sirt2-knockout mice and SIRT2 inhibitors, one study concluded that SIRT2 inhibited T-cell metabolism and impaired T-cell effector functions by deacetylating a number of metabolic enzymes. This conclusion was supported by the fact that SIRT2 was only increased in TILs from patients with NSCLC that responded partially to TIL therapy (38).

SIRT2 inhibitors. Recent studies have shown that inhibition of SIRT2 exhibits broad anticancer activity $(59,80)$. High expression of SIRT2 in NSCLC samples can be used to ensure the efficacy of SIRT2 inhibitors in NSCLC treatment. The existing SIRT2 inhibitors (AEM1 and AEM2) have shown p53-dependent proapoptotic activity in NSCLC (81). In addition, combination chemotherapy is a potentially promising approach used to enhance anticancer activity. The combination of SIRT2 inhibitors (AGK2 and Sirtinol) and the pyruvate dehydrogenase kinase inhibitor (dichloroacetic acid) was highly effective for inhibiting the proliferation of NSCLC cells (82). Bisnaphthalimidopropyl diaminodicyclohexylmethane is a polyamine derivative that inhibits growth and induces the apoptosis of NSCLC cancer cells. This anticancer effect is possibly mediated by SIRT2 inhibition (83). Moreover, inhibition of SIRT1 and SIRT2 by salermide, a reverse amide compound, leads to the upregulation of death receptor 5 through the activating transcription factor (ATF)4/ATF3/DNA damage inducible transcript 3 pathway in NSCLC, resulting in the apoptosis of human lung cancer cells (84). The anticancer effect of SIRT2 inhibitors in NSCLC provides a rational therapeutic strategy for NSCLC tumors with high expression of SIRT2. A schematic diagram of the biological functions of SIRT2 is shown in Fig. 3.

\section{Conclusion}

As aforementioned, NSCLC is a malignant cancer with a complicated etiology and poor prognosis, accounting for $\sim 80 \%$ of the total incidence of lung cancer. SIRT2 functions either as a tumor suppressor or as an oncogene in NSCLC, depending on the experimental conditions. It is notable that SIRT2 inhibitors exhibit a protective effect in patients with lung cancer 
and high SIRT2 expression, providing a theoretical basis for successful cancer therapy.

SIRT2 is mainly localized in the cytoplasm. However, it is also found in the nucleus and mitochondria. A secretome study performed in mouse macrophages revealed 775 proteins including SIRT2, which were reproducibly detected in the culture medium following lipopolysaccharide stimulation (85), suggesting a potential role of SIRT2 in the extracellular compartment. The applications of high-throughput liquid chromatography tandem mass spectrometry analysis have resulted in the identification of a large number of extracellular matrix and peripheral proteins that are acetylated in both healthy and diseased tissues (86-88). However, additional studies are required to assess whether the SIRT2 deacetylase activity in the extracellular tumor microenvironment can regulate tumorigenesis.

In conclusion, SIRT2 is implicated in a wide range of physiological and pathological processes via deacetylation or fatty deacylation of specific substrates. Dysregulation of SIRT2 is closely associated with NSCLC progression. SIRT2 exhibits both oncogenic and tumor suppressive functions depending on the cancer stage, cell molecular characteristics and experimental conditions. Therefore, despite the broad anticancer activity of SIRT2 inhibitors, extensive research is still required to validate the potential of SIRT2 as a target for NSCLC treatment.

\section{Acknowledgements}

Not applicable.

\section{Funding}

The present study was funded by the Priority Academic Program Development of Jiangsu Higher Education Institutions, as well as by a grant from the China Natural Science Foundation (grant no. 31801058).

\section{Availability of data and materials}

Not applicable.

\section{Authors' contributions}

$\mathrm{MZ}$ and MW searched the literature and wrote the manuscript. YEC and $\mathrm{CH}$ searched the literature and revised the manuscript. All authors have read and approved the final manuscript. Data authentication is not applicable.

\section{Ethics approval and consent to participate}

No applicable.

\section{Patient consent for publication}

Not applicable.

\section{Competing interests}

The authors declare that they have no competing interests.

\section{References}

1. Klar AJ, Fogel S and Macleod K: MAR1-a Regulator of the HMa and HMalpha Loci in Saccharomyces Cerevisiae. Genetics 93: 37-50, 1979.

2. Loo S and Rine J: Silencing and heritable domains of gene expression. Annu Rev Cell Dev Biol 11: 519-548, 1995.

3. Gottlieb S and Esposito RE: A new role for a yeast transcriptional silencer gene, SIR2, in regulation of recombination in ribosomal DNA. Cell 56: 771-776, 1989

4. Guarente L: Diverse and dynamic functions of the Sir silencing complex. Nat Genet 23: 281-285, 1999.

5. Tanny JC, Dowd GJ, Huang J, Hilz H and Moazed D: An enzymatic activity in the yeast Sir2 protein that is essential for gene silencing. Cell 99: 735-745, 1999

6. Tanner KG, Landry J, Sternglanz R and Denu JM: Silent information regulator 2 family of NAD-dependent histone/protein deacetylases generates a unique product, 1-O-acetyl-ADP-ribose. Proc Natl Acad Sci USA 97: 14178-14182, 2000.

7. Hirschey MD: Old enzymes, new tricks: Sirtuins are NAD(+)-dependent de-acylases. Cell Metab 14: 718-719, 2011.

8. Haigis MC, Mostoslavsky R, Haigis KM, Fahie K, Christodoulou DC, Murphy AJ, Valenzuela DM, Yancopoulos GD, Karow M, Blander G, et al: SIRT4 inhibits glutamate dehydrogenase and opposes the effects of calorie restriction in pancreatic beta cells. Cell 126: 941-954, 2006.

9. de Ruijter AJ, van Gennip AH, Caron HN, Kemp S and van Kuilenburg AB: Histone deacetylases (HDACs): Characterization of the classical HDAC family. Biochem J 370 (Pt 3): 737-749, 2003.

10. Feldman JL, Dittenhafer-Reed KE, Kudo N, Thelen JN, Ito A, Yoshida M and Denu JM: Kinetic and structural basis for acyl-group selectivity and NAD(+) dependence in sirtuin-catalyzed deacylation. Biochemistry 54: 3037-3050, 2015.

11. Maxwell MM, Tomkinson EM, Nobles J, Wizeman JW, Amore AM, Quinti L, Chopra V, Hersch SM and Kazantsev AG: The Sirtuin 2 microtubule deacetylase is an abundant neuronal protein that accumulates in the aging CNS. Hum Mol Genet 20: 3986-3996, 2011.

12. Rack JG, VanLinden MR, Lutter T, Aasland R and Ziegler M: Constitutive nuclear localization of an alternatively spliced sirtuin-2 isoform. J Mol Biol 426: 1677-1691, 2014.

13. Kudo N, Ito A, Arata M, Nakata A and Yoshida M: Identification of a novel small molecule that inhibits deacetylase but not defatty-acylase reaction catalysed by SIRT2. Philos Trans R Soc Lond B Biol Sci 373: 20170070, 2018.

14. Mei Z, Zhang X, Yi J, Huang J, He J and Tao Y: Sirtuins in metabolism, DNA repair and cancer. J Exp Clin Cancer Res 35: 182, 2016.

15. North BJ and Verdin E: Interphase nucleo-cytoplasmic shuttling and localization of SIRT2 during mitosis. PLoS One 2: e784, 2007.

16. Mathias RA, Greco TM, Oberstein A, Budayeva HG, Chakrabarti R, Rowland EA, Kang Y, Shenk T and Cristea IM: Sirtuin 4 is a lipoamidase regulating pyruvate dehydrogenase complex activity. Cell 159: 1615-1625, 2014

17. Tong Z, Wang M, Wang Y, Kim DD, Grenier JK, Cao J, Sadhukhan S, Hao Q and Lin H: SIRT7 Is an RNA-activated protein lysine deacylase. ACS Chem Biol 12: 300-310, 2017.

18. Eskandarian HA, Impens F, Nahori MA, Soubigou G, Coppée JY, Cossart P and Hamon MA: A role for SIRT2-dependent histone H3K18 deacetylation in bacterial infection. Science 341: 1238858, 2013

19. Cha Y, Han MJ, Cha HJ, Zoldan J, Burkart A, Jung JH, Jang Y, $\mathrm{Kim} \mathrm{CH}$, Jeong $\mathrm{HC}$, Kim BG, et al: Metabolic control of primed human pluripotent stem cell fate and function by the miR-200c-SIRT2 axis. Nat Cell Biol 19: 445-456, 2017.

20. Fiskus W, Coothankandaswamy V, Chen J, Ma H, Ha K, Saenz DT, Krieger SS, Mill CP, Sun B, Huang P, et al: SIRT2 deacetylates and inhibits the peroxidase activity of peroxiredoxin-1 to sensitize breast cancer cells to oxidant stress-inducing agents. Cancer Res 76: 5467-5478, 2016.

21. Rothgiesser KM, Erener S, Waibel S, Lüscher B and Hottiger MO: SIRT2 regulates NF-kappaB dependent gene expression through deacetylation of p65 Lys310. J Cell Sci 123 (Pt 24): 4251-4258, 2010.

22. Tsusaka T, Guo T, Yagura T, Inoue T, Yokode M, Inagaki N and Kondoh H: Deacetylation of phosphoglycerate mutase in its distinct central region by SIRT2 down-regulates its enzymatic activity. Genes Cells 19: 766-777, 2014. 
23. Sarikhani M, Mishra S, Desingu PA, Kotyada C, Wolfgeher D, Gupta MP, Singh M and Sundaresan NR: SIRT2 regulates oxidative stress-induced cell death through deacetylation of c-Jun $\mathrm{NH}_{2}$-terminal kinase. Cell Death Differ 25: 1638-1656, 2018.

24. Fabrizio P, Gattazzo C, Battistella L, Wei M, Cheng C, McGrew K and Longo VD: Sir2 blocks extreme life-span extension. Cell 123: 655-667, 2005.

25. Horio Y, Hayashi T, Kuno A and Kunimoto R: Cellular and molecular effects of sirtuins in health and disease. Clin Sci (Lond) 121: 191-203, 2011.

26. Michan S and Sinclair D: Sirtuins in mammals: Insights into their biological function. Biochem J 404: 1-13, 2007.

27. North BJ, Rosenberg MA, Jeganathan KB, Hafner AV, Michan S, Dai J, Baker DJ, Cen Y, Wu LE, Sauve AA, et al: SIRT2 induces the checkpoint kinase BubR1 to increase lifespan. EMBO J 33: 1438-1453, 2014

28. Dryden SC, Nahhas FA, Nowak JE, Goustin AS and Tainsky MA: Role for human SIRT2 NAD-dependent deacetylase activity in control of mitotic exit in the cell cycle. Mol Cell Biol 23: 3173-3185, 2003.

29. Vaquero A, Scher MB, Lee DH, Sutton A, Cheng HL, Alt FW, Serrano L, Sternglanz R and Reinberg D: SirT2 is a histone deacetylase with preference for histone $\mathrm{H} 4 \mathrm{Lys} 16$ during mitosis. Genes Dev 20: 1256-1261, 2006.

30. Serrano L, Martínez-Redondo P,Marazuela-Duque A, Vazquez BN, Dooley SJ, Voigt P, Beck DB, Kane-Goldsmith N, Tong Q, Rabanal RM, et al: The tumor suppressor SirT2 regulates cell cycle progression and genome stability by modulating the mitotic deposition of H4K20 methylation. Genes Dev 27: 639-653, 2013.

31. Kim HS, Vassilopoulos A, Wang RH, Lahusen T, Xiao Z, Xu X, Li C, Veenstra TD, Li B, Yu H, et al: SIRT2 maintains genome integrity and suppresses tumorigenesis through regulating APC/C activity. Cancer Cell 20: 487-499, 2011.

32. Chen G, Luo Y, Warncke K, Sun Y, Yu DS, Fu H, Behera M, Ramalingam SS, Doetsch PW, Duong DM, et al: Acetylation regulates ribonucleotide reductase activity and cancer cell growth. Nat Commun 10: 3213, 2019.

33. Gonfloni S, Iannizzotto V, Maiani E, Bellusci G, Ciccone S and Diederich M: P53 and Sirt1: Routes of metabolism and genome stability. Biochem Pharmacol 92: 149-156, 2014

34. Cha YI and Kim HS: Emerging role of sirtuins on tumorigenesis: Possible link between aging and cancer. BMB Rep 46: 429-438, 2013.

35. Li Y,Zhang M,Dorfman RG,Pan Y, Tang D, XuL,Zhao Z,Zhou Q, Zhou L, Wang Y, et al: SIRT2 promotes the migration and invasion of gastric cancer through RAS/ERK/JNK/MMP-9 pathway by increasing PEPCK1-Related metabolism. Neoplasia 20 745-756, 2018

36. Park SH, Ozden O, Liu G, Song HY, Zhu Y, Yan Y, Zou X, Kang HJ, Jiang H, Principe DR, et al: SIRT2-Mediated deacetylation and tetramerization of pyruvate kinase directs glycolysis and tumor growth. Cancer Res 76: 3802-3812, 2016.

37. Wang YP, Zhou LS, Zhao YZ, Wang SW, Chen LL, Liu LX, Ling ZQ, Hu FJ, Sun YP, Zhang JY, et al: Regulation of G6PD acetylation by SIRT2 and KAT9 modulates NADPH homeostasis and cell survival during oxidative stress. EMBO J 33: 1304-1320, 2014.

38. Hamaidi I, Zhang L, Kim N, Wang MH, Iclozan C, Fang B, Liu M, Koomen JM, Berglund AE, Yoder SJ, et al: Sirt2 inhibition enhances metabolic fitness and effector functions of Tumor-Reactive T Cells. Cell Metab 32: 420-436.e412, 2020.

39. Jing $H$, Zhang $X$, Wisner SA, Chen X, Spiegelman NA, Linder ME and Lin H: SIRT2 and lysine fatty acylation regulate the transforming activity of K-Ras4a. Elife 6: e32436, 2017.

40. Spiegelman NA, Zhang X, Jing H, Cao J, Kotliar IB, Aramsangtienchai $\mathrm{P}$, Wang $\mathrm{M}$, Tong $\mathrm{Z}$, Rosch $\mathrm{KM}$ and Lin $\mathrm{H}$ : SIRT2 and Lysine fatty acylation regulate the activity of RalB and cell migration. ACS Chem Biol 14: 2014-2023, 2019.

41. Kosciuk T, Price IR, Zhang X, Zhu C, Johnson KN, Zhang S, Halaby SL, Komaniecki GP, Yang M, DeHart CJ, et al: NMT1 and NMT2 are lysine myristoyltransferases regulating the ARF6 GTPase cycle. Nat Commun 11: 1067, 2020.

42. Chen G, Huang P and Hu C: The role of SIRT2 in cancer: A novel therapeutic target. Int J Cancer 147: 3297-3304, 2020.

43. Liu Y, Zhang Y, Zhu K, Chi S, Wang C and Xie A: Emerging role of Sirtuin 2 in Parkinson's disease. Front Aging Neurosci 11: $372,2020$.

44. Zhang Y, Anoopkumar-Dukie S, Arora D and Davey AK: Review of the anti-inflammatory effect of SIRT1 and SIRT2 modulators on neurodegenerative diseases. Eur J Pharmacol 867: 172847, 2020.
45. Reifenberger J, Reifenberger G, Liu L, James CD, Wechsler W and Collins VP: Molecular genetic analysis of oligodendroglial tumors shows preferential allelic deletions on 19q and 1p. Am J Pathol 145: 1175-1190, 1994.

46. Head PE, Zhang H, Bastien AJ, Koyen AE, Withers AE, Daddacha WB, Cheng X and Yu DS: Sirtuin 2 mutations in human cancers impair its function in genome maintenance. J Biol Chem 292: 9919-9931, 2017.

47. Wang B, Ye Y, Yang X, Liu B, Wang Z, Chen S, Jiang K, Zhang W, Jiang H, Mustonen H, et al: SIRT2-dependent IDH1 deacetylation inhibits colorectal cancer and liver metastases. EMBO Rep 21: e48183, 2020.

48. Carafa V, Altucci L and Nebbioso A: Dual tumor suppressor and tumor promoter action of sirtuins in determining malignant phenotype. Front Pharmacol 10: 38, 2019.

49. Li Z, Xie QR, Chen Z, Lu S and Xia W: Regulation of SIRT2 levels for human non-small cell lung cancer therapy. Lung Cancer 82: 9-15, 2013.

50. Grbesa I, Pajares MJ, Martínez-Terroba E, Agorreta J, Mikecin AM, Larráyoz M, Idoate MA, Gall-Troselj K, Pio R and Montuenga LM: Expression of sirtuin 1 and 2 is associated with poor prognosis in non-small cell lung cancer patients. PLoS One 10: e0124670, 2015.

51. Gao CX, Chen B, Xie HK, Han $\mathrm{CN}$ and Luo J: Immunohistochemistry and clinical value of sirtuin 2 in non-metastasized non-small cell lung cancer. J Thorac Dis 11: 3973-3979, 2019.

52. McGlynn LM, Zino S, MacDonald AI, Curle J, Reilly JE, Mohammed ZM, McMillan DC, Mallon E, Payne AP, Edwards J and Shiels PG: SIRT2: Tumour suppressor or tumour promoter in operable breast cancer? Eur J Cancer 50: 290-301, 2014.

53. Gong J, Wang H, Lou W, Wang G, Tao H, Wen H, Liu Y and Xie Q: Associations of sirtuins with clinicopathological parameters and prognosis in non-small cell lung cancer. Cancer Manag Res 10: 3341-3356, 2018.

54. Berggrund M, Enroth S, Lundberg M, Assarsson E, Stålberg K, Lindquist D, Hallmans G, Grankvist K, Olovsson $M$ and Gyllensten U: Identification of candidate plasma protein biomarkers for cervical cancer using the multiplex proximity extension assay. Mol Cell Proteomics 18: 735-743, 2019.

55. Inoue K, Mallakin A and Frazier DP: Dmpl and tumor suppression. Oncogene 26: 4329-4335, 2007.

56. Das C, Lucia MS, Hansen KC and Tyler JK: CBP/p300-mediated acetylation of histone H3 on lysine 56. Nature 459: 113-117, 2009.

57. Jin YH, Kim YJ, Kim DW, Baek KH, Kang BY, Yeo CY and Lee KY: Sirt2 interacts with 14-3-3 beta/gamma and down-regulates the activity of p53. Biochem Biophys Res Commun 368: 690-695, 2008

58. Zhao D, Zou SW, Liu Y, Zhou X, Mo Y, Wang P, Xu YH, Dong B, Xiong Y, Lei QY and Guan KL: Lysine-5 acetylation negatively regulates lactate dehydrogenase $\mathrm{A}$ and is decreased in pancreatic cancer. Cancer Cell 23: 464-476, 2013.

59. Jing H, Hu J, He B, Negrón Abril YL, Stupinski J, Weiser K, Carbonaro M, Chiang YL, Southard T, Giannakakou P, et al: A SIRT2-Selective inhibitor promotes c-Myc oncoprotein degradation and exhibits broad anticancer activity. Cancer Cell 29: 607, 2016.

60. Yang MH, Laurent G, Bause AS, Spang R, German N, Haigis MC and Haigis KM: HDAC6 and SIRT2 regulate the acetylation state and oncogenic activity of mutant K-RAS. Mol Cancer Res 11: 1072-1077, 2013

61. Spiegelman NA, Hong JY, Hu J, Jing H, Wang M, Price IR, Cao J, Yang M, Zhang X and Lin H: A Small-Molecule SIRT2 inhibitor that promotes K-Ras4a lysine fatty-acylation. ChemMedChem 14: 744-748, 2019.

62. Liu PY, Xu N, Malyukova A, Scarlett CJ, Sun YT, Zhang XD, Ling D, Su SP, Nelson C, Chang DK, et al: The histone deacetylase SIRT2 stabilizes Myc oncoproteins. Cell Death Differ 20: 503-514, 2013

63. Luo J, Bao YC, Ji XX, Chen B, Deng QF and Zhou SW: SPOP promotes SIRT2 degradation and suppresses non-small cell lung cancer cell growth. Biochem Biophys Res Commun 483: 880-884, 2017.

64. Xu Y, Li F, Lv L, Li T, Zhou X, Deng CX, Guan KL, Lei QY and Xiong Y: Oxidative stress activates SIRT2 to deacetylate and stimulate phosphoglycerate mutase. Cancer Res 74: 3630-3642, 2014.

65. Zhao Y, Yang J, Liao W, Liu X, Zhang H, Wang S, Wang D, Feng J, Yu L and Zhu WG: Cytosolic FoxO1 is essential for the induction of autophagy and tumour suppressor activity. Nat Cell Biol 12: 665-675, 2010 
66. Tang HX, Wang MY, Xiao W and Wen JW: SIRT2-Reverses Drug-Resistance of HL-60/A through autophagy mechanism. Zhongguo Shi Yan Xue Ye Xue Za Zhi 27: 409-414, 2019 (In Chinese)

67. Liu L, Yu L, Zeng C, Long H, Duan G, Yin G, Dai X and Lin Z: E3 Ubiquitin Ligase HRD1 promotes lung tumorigenesis by promoting sirtuin 2 ubiquitination and degradation. Mol Cel Biol 40: e00257-19, 2020

68. Zhu H, Hu Y, Zeng C, Chang L, Ge F, Wang W, Yan F, Zhao Q, Cao J, Ying M, et al: The SIRT2-mediated deacetylation of AKR1C1 is required for suppressing its pro-metastasis function in Non-small cell lung cancer. Theranostics 10: 2188-2200, 2020.

69. Lin R, Tao R, Gao X, Li T, Zhou X, Guan KL, Xiong Y and Lei QY: Acetylation stabilizes ATP-citrate lyase to promote lipid biosynthesis and tumor growth. Mol Cell 51: 506-518, 2013.

70. Mu N, Lei Y, Wang Y, Wang Y, Duan Q, Ma G, Liu X and Su L: Inhibition of SIRT1/2 upregulates HSPA5 acetylation and induces pro-survival autophagy via ATF4-DDIT4-mTORC1 axis in human lung cancer cells. Apoptosis 24: 798-811, 2019.

71. Wang L, Xu P, Xie X, Hu F, Jiang L, Hu R, Ding F, Xiao H and Zhang H: Down regulation of SIRT2 Reduced ASS induced NSCLC apoptosis through the release of autophagy components via exosomes. Front Cell Dev Biol 8: 601953, 2020.

72. Bajpe PK, Prahallad A, Horlings H, Nagtegaal I, Beijersbergen R and Bernards R: A chromatin modifier genetic screen identifies SIRT2 as a modulator of response to targeted therapies through the regulation of MEK kinase activity. Oncogene 34: 531-536, 2015.

73. Xu H, Li Y, Chen L, Wang C, Wang Q, Zhang H, Lin Y, Li Q and Pang T: SIRT2 mediates multidrug resistance in acute myelogenous leukemia cells via ERK1/2 signaling pathway. Int J Oncol 48: 613-623, 2016.

74. Zhang M, Du W, Acklin S, Jin S and Xia F: SIRT2 protects peripheral neurons from cisplatin-induced injury by enhancing nucleotide excision repair. J Clin Invest 130: 2953-2965, 2020

75. Zhao D, Mo Y, Li MT, Zou SW, Cheng ZL, Sun YP, Xiong Y, Guan KL and Lei QY: NOTCH-induced aldehyde dehydrogenase 1A1 deacetylation promotes breast cancer stem cells. J Clin Invest 124: 5453-5465, 2014.

76. Wei R, He D and Zhang X: Role of SIRT2 in regulation of stemness of cancer stem-like cells in renal cell carcinoma. Cell Physiol Biochem 49: 2348-2357, 2018.

77. Eramo A, Haas TL and De Maria R: Lung cancer stem cells: Tools and targets to fight lung cancer. Oncogene 29: 4625-4635, 2010.

78. Jiang F, Qiu Q, Khanna A, Todd NW, Deepak J, Xing L, Wang H, Liu Z, Su Y, Stass SA and Katz RL: Aldehyde dehydrogenase 1 is a tumor stem cell-associated marker in lung cancer. Mol Cancer Res 7: 330-338, 2009
79. Rotili D, Tarantino D, Nebbioso A, Paolini C, Huidobro C, Lara E, Mellini P, Lenoci A, Pezzi R, Botta G, et al: Discovery of salermide-related sirtuin inhibitors: Binding mode studies and antiproliferative effects in cancer cells including cancer stem cells. J Med Chem 55: 10937-10947, 2012.

80. Hu F, Sun X, Li G, Wu Q, Chen Y, Yang X, Luo X, Hu J and Wang G: Inhibition of SIRT2 limits tumour angiogenesis via inactivation of the STAT3/VEGFA signalling pathway. Cell Death Dis 10: 9, 2018.

81. Hoffmann G, Breitenbücher F, Schuler $M$ and Ehrenhofer-Murray AE: A novel sirtuin 2 (SIRT2) inhibitor with p53-dependent pro-apoptotic activity in non-small cell lung cancer. J Biol Chem 289: 5208-5216, 2014.

82. Ma W, Zhao X, Wang K, Liu J and Huang G: Dichloroacetic acid (DCA) synergizes with the SIRT2 inhibitor Sirtinol and AGK2 to enhance anti-tumor efficacy in non-small cell lung cancer. Cancer Biol Ther 19: 835-846, 2018.

83. Lima RT, Barron GA, Grabowska JA, Bermano G, Kaur S, Roy N, Vasconcelos MH and Lin PK: Cytotoxicity and cell death mechanisms induced by a novel bisnaphthalimidopropyl derivative against the NCI-H460 non-small lung cancer cell line. Anticancer Agents Med Chem 13: 414-421, 2013.

84. Liu G, Su L, Hao X, Zhong N, Zhong D, Singhal S and Liu X: Salermide up-regulates death receptor 5 expression through the ATF4-ATF3-CHOP axis and leads to apoptosis in human cancer cells. J Cell Mol Med 16: 1618-1628, 2012.

85. Meissner F, Scheltema RA, Mollenkopf HJ and Mann M: Direct proteomic quantification of the secretome of activated immune cells. Science 340: 475-478, 2013.

86. Lundby A, Lage K, Weinert BT, Bekker-Jensen DB, Secher A, Skovgaard T, Kelstrup CD, Dmytriyev A, Choudhary C, Lundby C and Olsen JV: Proteomic analysis of lysine acetylation sites in rat tissues reveals organ specificity and subcellular patterns. Cell Rep 2: 419-431, 2012.

87. Simon GM, Cheng J and Gordon JI: Quantitative assessment of the impact of the gut microbiota on lysine epsilon-acetylation of host proteins using gnotobiotic mice. Proc Natl Acad Sci USA 109: 11133-11138, 2012

88. Choudhary C, Kumar C, Gnad F, Nielsen ML, Rehman M, Walther TC, Olsen JV and Mann M: Lysine acetylation targets protein complexes and co-regulates major cellular functions. Science 325: 834-840, 2009. International (CC BY-NC-ND 4.0) License. 